\title{
The Association of Skinfold Anthropometric Measures, Body Composition and Disease Severity in Obese and Non-obese Fibromyalgia Patients: A Cross-sectional Study
}

\author{
Mehmet Onat ÇAKIT, ${ }^{1}$ Burcu Duyur ÇAKIT, ${ }^{2}$ Hakan GENÇ, ${ }^{2}$ Seçil PERVANE VURAL, ${ }^{2}$ \\ Hatice Rana ERDEM, ${ }^{3}$ Meryem SARAÇOĞLU, ${ }^{2}$ Aynur KARAGÖZ ${ }^{2}$ \\ ${ }^{1}$ Department of Family Medicine, Ankara Training and Research Hospital, Ankara, Turkey \\ ${ }^{2}$ Department of Physical Medicine and Rehabilitation, Ankara Training and Research Hospital, Ankara, Turkey \\ ${ }^{3}$ Department of Physical Medicine and Rehabilitation, Medical Faculty of Ahi Evran University, Kirşehir, Turkey
}

\begin{abstract}
Objectives: This study aims to determine the effects of obesity and obesity related anthropometric and body composition determiners on the severity of fibromyalgia syndrome (FS) and to compare obese, overweight and normoweight FS patients according to general health and psychological status.

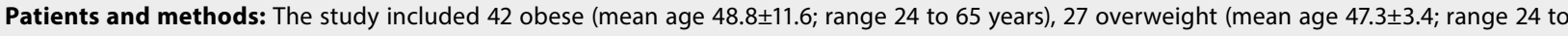
61 years) and 32 normoweight (mean age $47.1 \pm 7.8$ years; range 31 to 60 years) female FS patients. Widespread pain scores and symptom severity scores were noted. Pain pressure thresholds of tender points and control points were measured and total myalgic score (TMS) was calculated. The anthropometric assessments and skinfold measurements of all participants were recorded. Quality of life was evaluated by Health Assessment Questionnaire while psychological status was evaluated using Beck Depression Inventory.

Results: Control points, TMS values and hand grip strength values of obese FS patients were significantly lower, while disease duration, symptom severity, widespread pain scores, visual analog scale and Health Assessment Questionnaire scores were significantly higher than normoweight and overweight FS patients. Fat free mass, fat mass, body fat percentage and waist/hip ratio values were significantly higher in obese FS patients than overweight and normoweight FS patients ( $p<0.001$ for all values). Stepwise linear regression analysis showed that increased body mass index, decreased fat free mass $(\mathrm{R} 2=0.11)$ and increased disease duration $(\mathrm{R} 2=0.13)$ were associated with lower TMS.
\end{abstract}

Conclusion: We found that obesity had significant negative effects on pain, disease severity and quality of life in patients with FS.

Keywords: Body composition; disease severity; fibromyalgia syndrome; obesity; skinfold.

Fibromyalgia syndrome (FS) is a chronic pain syndrome which negatively affects the quality of life (QoL), characterized by widespread pain, stiffness, fatigue, sleep difficulties, increased pain sensitivity to pressure, psychological comorbidities and other symptoms. ${ }^{1}$ It was shown that especially physically inactive individuals and obese patients have an increased risk of developing FS. ${ }^{2}$

Obesity is characterized with additional fat-free mass (FFM) as well as fat mass (FM). Measurement of FM is more suitable than body mass index (BMI) in evaluating obesity. ${ }^{3}$ Several methods can be used for the assessment of body composition such as bioelectric impedance analysis, ultrasound, dual-energy X-ray absorptiometry or skinfold method. Skinfold method can be used as an accurate and convenient method to measure body composition in clinical setting. ${ }^{4}$ In previous studies, bioelectric impedance analysis method was used for the body composition analysis of patients with FS. 1,5,6 Ultrasound and skinfolds are validated to be accurate, portable and non-invasive methods in the assessment of FM in normoactive Turkish 
university students. ${ }^{7}$ To our knowledge, there is no study using skinfold method for measuring body composition in patients with FS.

Several studies showed a prevalence of overweight of about $20-30 \%$ and a prevalence of obesity of about $40-50 \%$ in patients with FS. ${ }^{2,8,9}$ Obesity can be considered an aggravating comorbid condition affecting negatively FS severity, global QoL, fatigue and physical function. $^{2}$ On the other hand, the associations between disease severity, body composition and obesity related skinfold anthropometric measurements have not been well-studied in obese FS patients. Therefore, in this study, we aimed to determine the effects of obesity and obesity related anthropometric and body composition determiners on the severity of FS and to compare obese, overweight and normoweight FS patients according to general health and psychological status.

\section{PATIENTS AND METHODS}

Between December 2013 and May 2014, a total of 70 females with FS and BMI of $<30 \mathrm{~kg} / \mathrm{m}^{2}$ and 100 females with FS and BMI of $\geq 30 \mathrm{~kg} / \mathrm{m}^{2}$ were screened at Ministry of Health Ankara Training and Research Hospital for this cross-sectional study. FS was diagnosed according to 2010 American College of Rheumatology preliminary diagnostic criteria. ${ }^{10}$ Individuals with active inflammatory/infectious disease, active psychiatric disease, and recent history of major trauma and active rheumatic disease were excluded. Forty-one patients refused to participate and 28 individuals who met the exclusion criteria were excluded. The World Health Organization (WHO) classification criteria were used to identify obese $\left(\mathrm{BMI}>30.0 \mathrm{~kg} / \mathrm{m}^{2}\right)$ and overweight patients $(\mathrm{BMI}=25-29.9) .{ }^{4}$ Finally, patients were grouped as 42 obese FS patients (mean age $48.8 \pm 11.6$; range 24 to 65 years), 27 overweight FS patients (mean age 47.3 \pm 3.4 ; range 24 to 61 years) and 32 normoweight FS patients (mean age $47.1 \pm 7.8$ years; range 31 to 60 years). In the study, overweight and normoweight FS patients, when used together, were referred to as non-obese FS patients. The study protocol was approved by the Ministry of Health Ankara Training and Research Hospital Ethics Committee. A written informed consent was obtained from each patient. The study was conducted in accordance with the principles of the Declaration of Helsinki.

Symptom severity score (SS) (0-12) and widespread pain index (WPI) (0-19) of FS patients were evaluated separately according to 2010 American College of Rheumatology preliminary diagnostic criteria. ${ }^{10}$ General pain severities (general visual analog scale [VAS]), neck and also low back pain (LBP) VAS were assessed separately using $0-10$ VAS $(0=$ no pain, $10=$ most severe pain).

Control and tender point pain pressure threshold (PPT) $\left(\mathrm{kg} / \mathrm{cm}^{2}\right)$ measurements were performed by the Fischer's tissue compliance meter (Pain Diagnostic \& Treatment, Italy) that can also be used as an algometer. ${ }^{11}$ During algometric studies, tender point (TP) measurements were performed according to 18 TPs defined by the American College of Rheumatology 1990 FS classification criteria, ${ }^{12}$ whereas the control point measurements were carried out bilaterally at the thumb nail and midpoint of the forearm volar surface. The individuals were informed that the examination was aimed at determining the pain threshold and not the pain tolerance. Then, pressure was raised at the rate of $1 \mathrm{~kg} / \mathrm{sec}$ until pain or discomfort occurred; the minimum force that caused pain was termed the PPT. Points that were painful with less than $4 \mathrm{~kg} / \mathrm{cm}^{2}$ pressure were accepted as TP. The severity of FS was determined with total myalgic score (TMS) and control point score. The sum of the PPTs of the 22 points (18 TPs and four control points) was calculated as TMS $\left(\mathrm{kg} / \mathrm{cm}^{2}\right)$ and the sum of the PPTs of the control points was termed as control point score $\left(\mathrm{kg} / \mathrm{cm}^{2}\right){ }^{13}$

Health Assessment Questionnaire (HAQ), which evaluates eight activities of daily living (dressing and grooming, arising, eating, walking, hygiene, reaching, grip and activities such as running errands), was used to assess functional difficulties in 0-3 scale. ${ }^{14}$

Isometric hand grip strength measurements were determined with Jamar hand dynamometer (Preston Co., Jackson, MI, USA). Three trials were performed for each measurement (with a 15-sec interval between each short grip strength measurement) and the means were recorded. 
Psychological status was utilized using Beck Depression Inventory in all groups. ${ }^{15}$ Waist circumference (WC) was determined using a spring scale at the end of gentle expiration at the level midway between the lower rib margin and the iliac crest (cut-off points for cardiovascular disease risk were $102 \mathrm{~cm}$ for males and 88 for females as defined by the criteria of WHO). Hip circumference was determined at the largest posterior extension of the buttocks. Waist hip ratio (WHR) was calculated by dividing these two values (cut-off points for cardiovascular disease risk was 1.0 in males and 0.85 in females as defined by the criteria of WHO). ${ }^{16}$ WC and WHR were used to assess body fat distribution and specifically as indicators of intraabdominal or visceral fat mass.

The amount and dispersion of body fat were evaluated by measuring the thickness of subcutaneous adipose tissue. For skinfold anthropometry, triple measurements were taken in four standard sites: biceps and triceps (limb), subscapular and suprailiac skinfolds (trunk) using a caliper (Lafayette Instrument Co. Indiana 47903). The sum of four skinfolds (triceps, biceps, subscapular and suprailiac) was used to calculate body density. Body density was estimated according to the Durnin and Womersley method, ${ }^{17}$ and FM calculated with the Siri's equation.

\section{Statistical analysis}

All statistical analyses were performed using SPSS for Windows version 15.0 software package (SPSS Inc., Chicago, IL, USA). A level of $p<0.05$ was considered statistically significant. One-way analysis of variance was conducted on three patient groups. Bonferroni post-hoc tests were conducted when analysis of variance showed significant effects. The distribution of categorical variables in groups was compared using Pearson Chi-square test. Pearson correlation analysis was conducted to determine the relationships between BMI and disease severity parameters. Results were reported as mean \pm standard deviation and median (minimum-maximum) when appropriate.

\section{RESULTS}

There was no difference between the groups in terms of mean age ( $>0.05)$. Disease duration was higher in obese FS patients than non-obese FS patients $(p<0.05)$. Grip strength values were lower in obese FS patients than non-obese FS patients (Table 1).

Symptom severity and WPI scores of obese FS patients were higher than non-obese FS patients. Neck pain VAS was higher in obese FS patients than non-obese FS patients. LBP VAS and general VAS values were higher in obeseand overweight FS patients than normoweight FS patients (Table 1).

Mean control point score and TMS values of obese FS patients were lower than non-obese FS patients. The mean number of TP was higher in obese FS patients than non-obese FS patients. The mean value of number of TP was not different among groups ( $p>0.05)$ The mean value of HAQ was higher in obese FS patients than non-obese FS patients but there was no difference among groups regarding Beck Depression Inventory scores ( $p>0.05)$ (Table 1).

The mean WC and WHR values were different among groups, according to the cut-off points for relative risk defined by WHO, cardiovascular disease risk determined by WHR (all $p<0.001$ ) and WC (all $\mathrm{p}<0.001$ ) in obese, normoweight and overweight FS patients (Table 2).

Fat mass and body fat percentage were higher in obese FS patients than non-obese FS patients $(p<0.001)$ (Table 2). Correlation analyses revealed positive correlations between $\mathrm{BMI}$ and SS $(r=0.339, p=0.001)$, WPI $(r=0.688, p<0.001)$, and number of tender points (NTP) $(r=0.426$, $\mathrm{p}<0.001)$ values and negative correlation with TMS $(\mathrm{r}=-0.354, \mathrm{p}<0.001)$.

Stepwise linear regression analyses were conducted to identify the affecting factors for disease severity (TMS, SS, WPI). Regression analysis showed that increased body fat percentage $\left(R^{2}=0.53\right)$ and increased VAS (general pain) $\left(\mathrm{R}^{2}=0.20\right)$ were associated with higher WPI. Body fat percentage was found to be a more prominent factor on WPI. A total of $73 \%$ variance was explained with body fat percentage and VAS on WPI.

\section{DISCUSSION}

In this study, we evaluated the effects of obesity and obesity related skinfold anthropometric and 


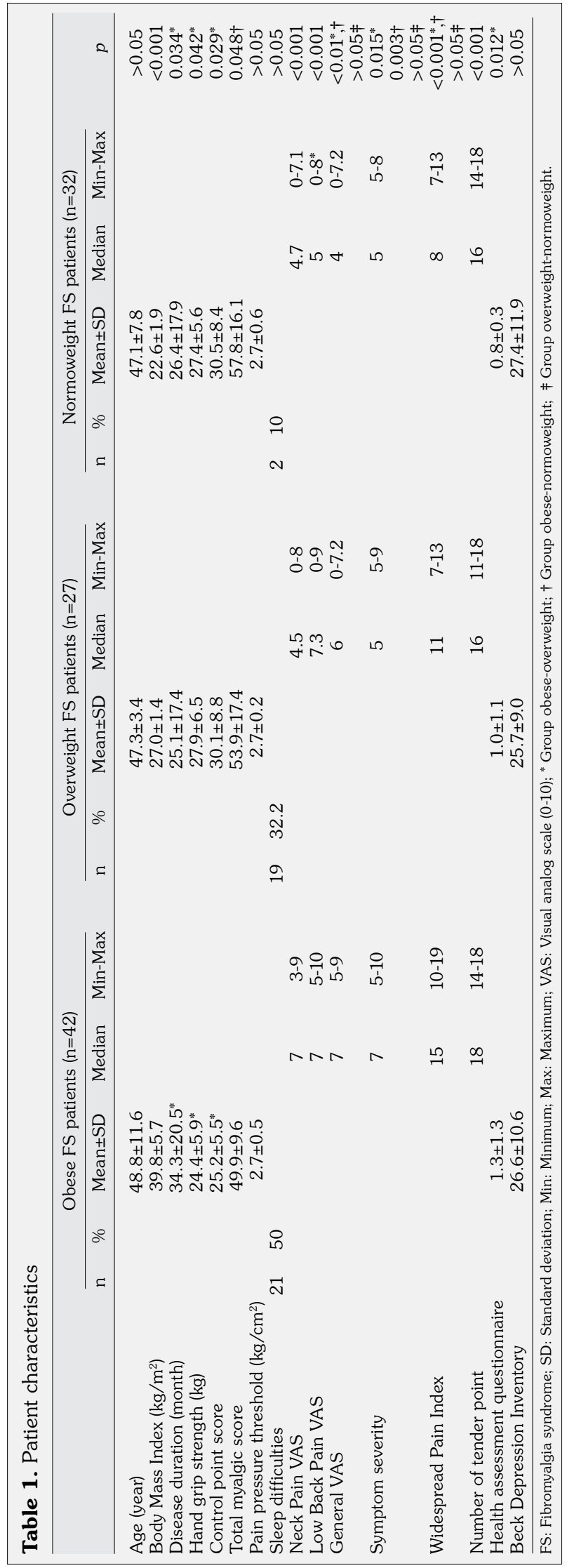

body composition determiners on the severity of FS and investigated the effect of FS on general health and psychological status. Clinical course of FS is more serious in obese FS patients than overweight and normoweight FS patients. BMI were found related with disease severity parameters (NTP, SS and WPI) and PPT values.

In several studies, a relationship between BMI and nonspecific LBP, other forms of chronic pain and headaches has been reported. The literature shows that obese people have more musculoskeletal pain and physical dysfunction than people with normoweight. ${ }^{8}$ McKendall and Haier ${ }^{18}$ found diminished mechanical pain thresholds in healthy obese individuals. Overweight category was also found related with an increased risk for cardiovascular disease in both sexes, LBP and poor QoL in females. ${ }^{19}$ We observed that chronic neck pain and LBP were frequent in our obese and non-obese FS patients. Moreover, in overweight FS patients, LBP and general VAS and TMS values were higher than normoweight FS patients. Our obese and overweight FS patients had increased cardiovascular risk which was determined with WHR. However, we did not find any difference among groups according to HAQ values.

Okifuji et al. ${ }^{20}$ found that the heightened pain sensitivity to pain TPs in obese FS patients appeared especially in the lower body areas. In addition to centrally modulated sensitivity, the pain sensitivity of obese FS patients may also be affected by the mechanical loads of having to carry extra weight. So, in our study, disease duration was found higher in obese FS patients than overweight and normoweight FS patients. We thought that disease duration could be augmented via obesity related noxious stimuli from the joints and muscles and may be potentiated by sensitivity to noxious stimuli via obesity-related alteration in the endocrine and opioid system.

The easiest explanation for the high prevalence of obesity in FS could be inadequate physical activity. ${ }^{21}$ Yunus et al. ${ }^{9}$ found that most FS patients are overweight, likely due to physical inactivity. Reduced hand grip strengths and QoL levels of our obese FS patients also indicated that obese FS patients had insufficient physical activity. So, encouraging these patients to physical activity may be essential for increasing QoL levels. 


\begin{tabular}{|c|c|c|c|c|c|c|c|c|c|c|}
\hline & \multicolumn{3}{|c|}{$\begin{array}{l}\text { Obese FS patients } \\
\qquad(\mathrm{n}=42)\end{array}$} & \multicolumn{3}{|c|}{$\begin{array}{l}\text { Overweight FS patients } \\
\qquad(\mathrm{n}=27)\end{array}$} & \multicolumn{3}{|c|}{$\begin{array}{l}\text { Normoweight FS patients } \\
\qquad(\mathrm{n}=32)\end{array}$} & \multirow[b]{2}{*}{$p$} \\
\hline & $\mathrm{n}$ & $\%$ & Mean \pm SD & $\mathrm{n}$ & $\%$ & Mean \pm SD & $\mathrm{n}$ & $\%$ & Mean \pm SD & \\
\hline Waist circumference $(\mathrm{cm})$ & & & $124.1 \pm 10.1$ & & & $90.7 \pm 7.5$ & & & $78.6 \pm 6.8$ & $<0.001$ \\
\hline No risk $(<80)$ & - & - & & 2 & 7.4 & & 18 & 56.3 & & \\
\hline Medium risk (80-87) & 1 & - & & 8 & 29.6 & & 12 & 37.5 & & $<0.001^{*}$ \\
\hline High risk $(>88)$ & 41 & - & & 17 & 63 & & 2 & 6.3 & & \\
\hline Hip circumference $(\mathrm{cm})$ & & & $126.6 \pm 8.1$ & & & $100.0 \pm 5.0$ & & & $99.2 \pm 6.2$ & $<0.001 \dagger, \ddagger$ \\
\hline Waist/Hip Ratio & & & $0.9 \pm 0.1$ & & & $0.9 \pm 0.09$ & & & $0.7 \pm 0.1$ & $<0.001$ \\
\hline No risk $(<0.80)$ & - & - & & 2 & & & 18 & & & \\
\hline Medium risk (0.81-0.85) & 4 & - & & 6 & & & 10 & & & $<0.001^{*}$ \\
\hline High risk (>0.85) & 41 & - & & 19 & & & 4 & & & \\
\hline Fat free mass & & & $50.7 \pm 7.9$ & & & $46.2 \pm 3.0$ & & & $46.6 \pm 2.5$ & $0.005 \dagger$ \\
\hline Fat mass & & & $46.5 \pm 8.9$ & & & $27.8 \pm 1.4$ & & & $23.1 \pm 1.9$ & $<0.001$ \\
\hline Body fat percentage & & & $49.1 \pm 2.8$ & & & $35.3 \pm 1.2$ & & & $30.8 \pm 1.3$ & $<0.001$ \\
\hline
\end{tabular}

Increased physical activity combined with weight loss can improve many adversities in the lives of these patients. Correspondingly, Shapiro et al. ${ }^{22}$ evaluated the effects of behavioral weight loss treatment on FS symptoms. At the end of the study, patients showed significant improvement in measures of depression (Beck Depression Inventory), pain, and anxiety, body satisfaction, and QoL. Similarly, Saber et al. ${ }^{23}$ investigated the effect of weight loss in FS by laparoscopic Rouxen-Y gastric bypass. After the surgery, the mean BMI was significantly reduced and there was a significant improvement in pain and NTP.

Furthermore, Yunus et al. ${ }^{9}$ evaluated 211 female patients with FS. In their study, HAQ scores were significantly correlated with BMI, while fatigue and NTP showed a trend of significance. Neumann et al. ${ }^{24}$ evaluated the relationship between $\mathrm{BMI}$ and tenderness measurement, QoL and physical functioning in 100 FS patients. In this study, BMI was negatively related with QoL and tenderness threshold and positively related with physical functioning and tender point count. In our study, we found that QoL assessments were poor in obese FS patients and obese FS patients displayed higher pain sensitivity. FS is also a syndrome of central sensitization and augmentation that results in widespread musculoskeletal tenderness and pain. Tenderness in FS is related to central sensitization with amplification of nociception, resulting in a broad array of stimuli perceived as being more painful among FS patients than healthy controls. This is a basic abnormality that is very likely related to the cause of FS. ${ }^{8,25}$ Therefore, we found that TMS was lower in obese FS patients than overweight and normoweight FS patients. Also, we found that WPI was found associated with body fat percentage. It is obvious that generalized pain sensitivity is associated with body fat percentage and increased disease duration may augment central sensitization. Another factor contributing to this augmentation may be the chronic pain emerging from excessive weight load on joints and soft tissues in obesity.

To our knowledge, three studies have been conducted to evaluate body composition in patients with FS. Arranz et al. ${ }^{1}$ directly evaluated the relationship between BMI, FM and FFM with short form-36 QoL scores in FS patients. They found that BMI had a negative correlation with emotional role, FM with pain and FFM almost with all scores but specifically with emotional role, vitality and physical role. Collado-Mateo et al. ${ }^{5}$ investigated fear of falling in FS patients. They also evaluated body composition in FS patients. They found that body composition and BMI were not different between FS and healthy controls. Lowe et al. ${ }^{6}$ evaluated the resting metabolic rates in FS patients. They also investigated the FM, FFM and body fat percentage parameters. They did not find any difference between FS patients and controls on anthropometric measures with the exception of basal body temperature and they also showed that resting metabolic rate values did not correlate with any measure of FS status. They also stated that a low resting metabolic rate is a major risk factor for obesity. In all 
studies mentioned above, body composition was measured using bioelectric impedance analyzer in patients with FS. To the best of our knowledge, ours is the first study using skinfold method for determining body composition in FS. We think that skinfold method is a simple, cheap and useful method in daily clinical practice.

A large, worldwide INTERHEART study (effect of potentially modifiable risk factors associated with myocardial infarction in 52 countries) suggests that WHR may provide the best anthropometric measure to estimate abdominal fat that predicts cardiovascular morbidity. ${ }^{26}$ Loevinger et al. $^{27}$ evaluated the metabolic syndrome in females with chronic pain. In their study, FS was found related with larger WC and higher WHR than healthy controls. They found that females with chronic pain from fibromyalgia are at increased risk for metabolic syndrome. Metabolic syndrome indicated risk for cardiovascular disease, diabetes mellitus and all cause of mortality. Similarly, we found that obese or non-obese FS patients had higher cardiovascular risk than healthy control according to WHR and body fat percentage values.

One of the limitations of our study was its small sample size. Another limitation was the lack of evaluating body composition with ultrasonography, dual-energy X-ray absorptiometry or bioimpedance analysis. However, we think that skinfold method is convenient for determining body composition in FS and can be used in clinical practice efficiently.

In conclusion, overweight and obesity are substantial health problems coexisting with FS. Our results showed that obesity increased disease severity in patients with FS. Obese patients with FS had poor QoL. Additionally, long disease duration may be responsible for the poor outcome in obese FS patients with FS. Thus, we think that obesity should be treated and physical activity should be encouraged in the early stages of obesity for a healthy life outcome in patients with FS.

\section{Declaration of conflicting interests}

The authors declared no conflicts of interest with respect to the authorship and/or publication of this article.

\section{Funding}

The authors received no financial support for the research and/or authorship of this article.

\section{REFERENCES}

1. Arranz L, Canela MA, Rafecas M. Relationship between body mass index, fat mass and lean mass with SF-36 quality of life scores in a group of fibromyalgia patients. Rheumatol Int 2012;32:3605-11.

2. Okifuji A, Bradshaw DH, Olson C. Evaluating obesity in fibromyalgia: neuroendocrine biomarkers, symptoms, and functions. Clin Rheumatol 2009;28:475-8.

3. Frankenfield DC, Rowe WA, Cooney RN, Smith JS, Becker D. Limits of body mass index to detect obesity and predict body composition. Nutrition 2001;17:26-30.

4. WHO. Obesity: preventing and managing the global epidemic. Report of a WHO Consultation. WHO Technical Report Series 894. Geneva: World Health Organization; 2000.

5. Collado-Mateo D, Gallego-Diaz JM, Adsuar JC, Domínguez-Muñoz FJ, Olivares PR, Gusi N. Fear of Falling in Women with Fibromyalgia and Its Relation with Number of Falls and Balance Performance. Biomed Res Int 2015;2015:589014.

6. Lowe JC, Yellin J, Honeyman-Lowe G. Female fibromyalgia patients: lower resting metabolic rates than matched healthy controls. Med Sci Monit 2006;12:282-9.

7. Duz S, Kocak M, Korkusuz F. Evaluation of body composition using three different methods compared to dual-energy X-ray absorptiometry. Eur J Sport Sci 2009;9:181-90.

8. Ursini F, Naty S, Grembiale RD. Fibromyalgia and obesity: the hidden link. Rheumatol Int 2011;31:1403-8.

9. Yunus MB, Arslan S, Aldag JC. Relationship between body mass index and fibromyalgia features. Scand $\mathrm{J}$ Rheumatol 2002;31:27-31.

10. Wolfe F, Clauw DJ, Fitzcharles MA, Goldenberg DL, Katz RS, Mease P, et al. The American College of Rheumatology preliminary diagnostic criteria for fibromyalgia and measurement of symptom severity. Arthritis Care Res (Hoboken) 2010;62:600-10.

11. Fischer AA. Tissue compliance meter for objective, quantitative documentation of soft tissue consistency and pathology. Arch Phys Med Rehabil 1987;68:122-5.

12. Wolfe F, Smythe HA, Yunus MB, Bennett RM, Bombardier C, Goldenberg DL, et al. The American College of Rheumatology 1990 Criteria for the Classification of Fibromyalgia. Report of the Multicenter Criteria Committee. Arthritis Rheum 1990;33:160-72.

13. Genc H, Nacir B, Duyur Cakit B, Saracoglu M, Erdem HR. The effects of coexisting fibromyalgia syndrome 
on pain intensity, disability, and treatment outcome in patients with chronic lateral epicondylitis. Pain Med 2012;13:270-80.

14. Fries JF, Spitz P, Kraines RG, Holman HR. Measurement of patient outcome in arthritis. Arthritis Rheum 1980;23:137-45.

15. Beck AT, Steer RA, Garbin GM. Psychometric properties of the Beck Depression Inventory: twenty-five years of evaluation. Clin Psychol Rev 1988;8:77-100.

16. WHO consultation on Obesity. Obesity: Preventing and Managing the Global Epidemic. Geneva, Switzerland: Division of Noncommunicable Diseases Programme of Nutrition. Family and Reproductive Health. World Health Organization; 1998.

17. Durnin JV, Womersley J. Body fat assessed from total body density and its estimation from skinfold thickness: measurements on 481 men and women aged from 16 to 72 years. Br J Nutr 1974;32:77-97.

18. McKendall MJ, Haier RJ. Pain sensitivity and obesity. Psychiatry Res 1983;8:119-25.

19. Lean ME, Han TS, Seidell JC. Impairment of health and quality of life using new US federal guidelines for the identification of obesity. Arch Intern Med 1999;159:837-43.

20. Okifuji A, Donaldson GW, Barck L, Fine PG. Relationship between fibromyalgia and obesity in pain, function, mood, and sleep. J Pain 2010;11:1329-37.
21. Henriksson CM. Longterm effects of fibromyalgia on everyday life. A study of 56 patients. Scand J Rheumatol 1994;23:36-41.

22. Shapiro JR, Anderson DA, Danoff-Burg S. A pilot study of the effects of behavioral weight loss treatment on fibromyalgia symptoms. J Psychosom Res 2005;59:275-82.

23. Saber AA, Boros MJ, Mancl T, Elgamal MH, Song $\mathrm{S}$, Wisadrattanapong $\mathrm{T}$. The effect of laparoscopic Roux-en-Y gastric bypass on fibromyalgia. Obes Surg 2008;18:652-5.

24. Neumann L, Lerner E, Glazer Y, Bolotin A, Shefer A, Buskila D. A cross-sectional study of the relationship between body mass index and clinical characteristics, tenderness measures, quality of life, and physical functioning in fibromyalgia patients. Clin Rheumatol 2008;27:1543-7.

25. Cakit BD, Taskin S, Nacir B, Unlu I, Genc H, Erdem HR. Comorbidity of fibromyalgia and cervical myofascial pain syndrome. Clin Rheumatol 2010;29:405-11.

26. Yusuf S, Hawken S, Ounpuu S, Bautista L, Franzosi MG, Commerford $\mathrm{P}$, et al. Obesity and the risk of myocardial infarction in 27,000 participants from 52 countries: a case-control study. Lancet 2005;366:1640-9.

27. Loevinger BL, Muller D, Alonso C, Coe CL. Metabolic syndrome in women with chronic pain. Metabolism 2007;56:87-93. 